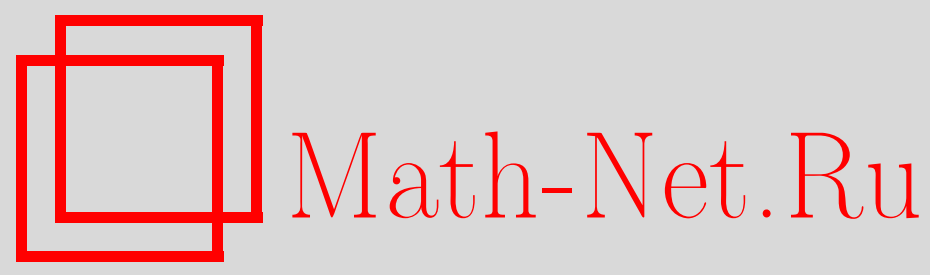

Н. М. Николов, Непрерывность и граничное поведение метрики Каратеодори, Матем. заметки, 2000, том 67, выпуск 2, 230-240

DOI: https://doi.org/10.4213/mzm831

Использование Общероссийского математического портала Math-Net.Ru подразумевает, что вы прочитали и согласны с пользовательским соглашением http://www . mathnet.ru/rus/agreement

Параметры загрузки:

IP: 18.208 .226 .222

26 апреля 2023 г., 10:08:53

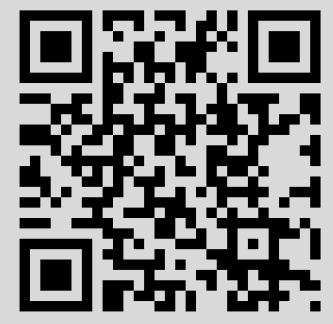


тОм 67 вЫПУСК 2 фЕВРАЛЬ 2000

УДК 517

\section{НЕПРЕРЫВНОСТЬ И ГРАНИЧНОЕ ПОВЕДЕНИЕ МЕТРИКИ КАРАТЕОДОРИ}

\section{Н. Николов}

Основной целью этой статьи является изучение граничного поведения метрик Каратеодори высшего порядка, сингулярной метрики Каратеодори и метрики Азукава вблизи $h$-растяжимой граничной точки ограниченной гладкой псевдовьпуклой области в $\mathbb{C}^{n}$.

Библиографйл: 10 названий.

1. Введение. В этой статье изучаются непрерывность и граничное поведение метрик Каратеодори высшего порядка, сингулярной метрики Каратеодори и метрики Азукава областей в $\mathbb{C}^{n}$.

Известно, что метрики Каратеодори высшего порядка всегда полунепрерывны сверху, но не обязаны быть непрерывны (за исключением стандартной) [1]. М. Ярницки и П. Пфлуг [1] доказали, что в ограниченных областях эти метрики непрерьвны. В настоящей статье этот результат обобщается (теорема 1) и доказывается непрерьвность метрик Каратеодори высшего порядка произвольной области в $\mathbb{C}^{n}$ в точках, где стандартная метрика Каратеодори положительна.

В теоремах 2 и 3 устанавливаются стабильность и непрерьвность сингулярной метрики Каратеодори строго гипервыпуклых областей [2] и модельных областей [3] соответственно. Из этих теорем и результата Ст. Нивоша [2] следует, что сингулярная метрика Каратеодори и метрика Азукава в таких областях совпадают (следствия 2 и 3 ). Отметим, что непрерьвность метрики Азукава для одного класса областей, включающего гипервыпуклые области, была доказана недавно Вл. Звонеком [4].

При помощи теорем 1 и 3 выводятся (теорема 4) точные некасательные пределы метрик Каратеодори высшего порядка, сингулярной метрики Каратеодори и метрики Азукава в $h$-растяжимой граничной точке гладкой ограниченной псевдовыпуклой области в $\mathbb{C}^{n}$. Случай стандартной метрики Каратеодори рассмотрен автором в [5].

2. Определения и формулировки результатов. Пусть $D$ - область в $\mathbb{C}^{n}$. Обозначим через $\operatorname{Hol}(D, \Delta)$ пространство всех голоморфных отображений из $D$ в единичный круг $\Delta$ в $\mathbb{C}$. Для каждого $k \in \mathbb{N}$ определим $k$-ю метрику Каратеодори следующим образом (см. [1]):

$$
C_{D}^{(k)}(z, X)=\sup \left\{\left|f_{(k)}(z) X\right|: f \in \operatorname{Hol}(D, \Delta), \operatorname{ord}_{z} f \geqslant k\right\}
$$


где $f_{(k)}(z) X=\sum_{|\alpha|=k}(1 / \alpha !) D^{\alpha} f(z) X^{\alpha}, \operatorname{a~ord}_{z} f$ означает порядок нуля $f$ в $z$. Отметим, что $C_{D}^{(1)}(z, X)$ - стандартная метрика Каратеодори. Легко видеть также, что

$$
\left(C_{D}^{(k)}(z, X)\right)^{k}\left(C_{D}^{(l)}(z, X)\right)^{l} \leqslant\left(C_{D}^{(k+l)}(z, X)\right)^{k+l} \quad \forall k, l \in \mathbb{N} .
$$

Это означает, что существует следующий предел:

$$
\lim _{k \rightarrow \infty} C_{D}^{(k)}(z, X)=: C_{D}^{\infty}(z, X) .
$$

Он назьвается сингулярной метрикой Каратеодори.

Функиия Грина и метрика Азукава определяются соответственно как

$$
\begin{gathered}
g_{D}(z, w)=\sup \{u(w): u-\text { отрищательная плюрисубгармоническая } \\
\text { функция в } \left.D \text { и } u(v) \leqslant \log \|v-z\|+O_{u}(1)\right\}, \\
A_{D}(z, X)=\limsup _{\lambda \rightarrow 0, \lambda \neq 0} \frac{\exp g(z, z+\lambda X)}{|\lambda|} .
\end{gathered}
$$

Отметим, что эти две функции полунепрерывны сверху по обеим переменным [6]. Кроме того, имеют место следующие неравенства:

$$
C_{D}^{(k)}(z, X) \leqslant C_{D}^{\infty}(z, X) \leqslant A_{D}(z, X)
$$

Область $D$ называется строго гипервыпуклой, если существуют другая область $D^{\prime} \ni D$ и плюрисубгармоническая функция $\varphi$ в $D^{\prime}$ такие, что $\lim _{z \rightarrow \partial D} \varphi(z)=0$ и $D=\left\{z \in D^{\prime}: \varphi(z)<0\right\}$.

Tеорема 1. Пусть $D-$ область в $\mathbb{C}^{n}$, а точка $z_{0} \in D$ такова, ито $C_{D}^{(1)}\left(z_{0}, X\right)>0$ $\forall X \in \mathbb{C}^{n} \backslash\{0\}$. Тогда для кажсдого $k \in \mathbb{N}$ существует постоянная $C>0$ и окрестность $U \subset D$ точки $z_{0}$ такие, что

$$
\left|C_{D}^{(k)}(z, X)-C_{D}^{(k)}(w, Y)\right| \leqslant C(\|X-Y\|+(\|X\|+\|Y\|)\|z-w\|)
$$

$\forall z, w \in U, \forall X, Y \in \mathbb{C}^{n}$.

Отметим, что для произвольной области $D$ функция $C_{D}^{(k)}(z, \cdot)$ непрерьвна и логарифм-плюрисубгармонична, но функция $C_{D}^{(k)}(\cdot, X)$ необязана быть непрерывной $(k \geqslant 2)$.

Из теоремы 1 непосредственно вытекает

СлЕДСТВИЕ 1. При условиях теоремы 1 сингулярная метрика Каратеодори $C_{D}^{\infty}(z, X)$ полунепрерывна снизу в $U \times \mathbb{C}^{n}$.

ТЕОрема 2. Пусть $D$ - строго гипервыпуклая область в $\mathbb{C}^{n}, a\left\{D_{j}\right\}_{j=1}^{\infty}-$ последовательность областей такая, что

$$
\lim _{j \rightarrow \infty} h\left(\partial D_{j}, \partial D\right)=0
$$

әде $h$ обозначает расстояние Хаусдорфа. Если $D_{j} \times \mathbb{C}^{n} \ni\left(z_{j}, X_{j}\right) \rightarrow(z, X) \in D \times \mathbb{C}^{n}$, mo

$$
\lim _{j \rightarrow \infty} C_{D_{j}}^{\infty}\left(z_{j}, X_{j}\right)=C_{D}^{\infty}(z, X) .
$$


СлЕДСтвИЕ 2. Сингулярная метрика Каратеодори строго гипервыпуклой области в $\mathbb{C}^{n}$ непрерывна и совпадает с метрикой Азукава.

Кроме того, при условиях теоремы 2 имеем

$$
\lim _{j \rightarrow \infty} A_{D_{j}}\left(z_{j}, X_{j}\right)=A_{D}(z, X) .
$$

Перед тем как изложить следующие результаты, напомним понятие $h$-растяжимой точки [3] (или полурегулярной точки в терминологии [7]). Пусть $D$ - область в $\mathbb{C}^{n}$ и $p \in \partial D$ - гладкая псевдовьпуклая граничная точка конечного типа с мультитипом Катлина $M=\left(m_{1}, m_{2}, \ldots, m_{n}\right)$ и $q$-типом Д'Анджело $\Delta_{q}$. Тогда $p$ называется $h$-растяжимой точкой, если $\Delta_{q}=m_{n+1-q}$ для $1 \leqslant q \leqslant n$.

Согласно [3] существует такая локальная система голоморфных координат $z=$ $\left(z_{1}, z^{\prime}\right)$, что в них $p=0$ и вблизи $p=0$ область $D$ может быть задана с помощью определяющей функции $r$ вида

$$
r(z)=\operatorname{Re} z_{1}+P\left(z^{\prime}\right)-R_{1}(z)+R_{2}(z) .
$$

Здесь $P$ - плюрисубгармонический полином, не имеющий гармонических составляющих и такой, что

$$
\begin{gathered}
P\left(t^{1 / m_{2}} z_{2}, \ldots, t^{1 / m_{n}} z_{n}\right)=t P\left(z^{\prime}\right) \quad \forall t>0, \quad z^{\prime} \in \mathbb{C}^{n-1} \\
0 \leqslant R_{1}(z) \leqslant C\left(\operatorname{Im} z_{1}\right)^{2}, \quad\left|R_{2}(z)\right| \leqslant C\left(\left(\sigma\left(z^{\prime}\right)\right)^{1+\gamma}\right.
\end{gathered}
$$

для $\sigma\left(z^{\prime}\right)=\sum_{j=2}^{n}\left|z_{j}\right|^{m_{j}}$ и каких-то положительных постоянных $C$ и $\gamma$.

Для каждого $\varepsilon \in \mathbb{R}$ положим

$$
E_{\varepsilon}=\left\{z \in \mathbb{C}^{n}: \operatorname{Re} z_{1}+P\left(z^{\prime}\right)+\varepsilon \sigma\left(z^{\prime}\right)<0\right\}
$$

Неограниченная область $E:=E_{0}$ назьвается моделью для $D$ в $p$. Отметим, что граничная точка $p \in \partial D$ является $h$-растяжимой тогда, когда соответствующая модель $E$ - область конечного типа (т.е. все ее граничные точки конечного типа) [3], [7].

Положим $S_{R}=\left\{z \in \mathbb{C}^{n}: \sigma(z):=\left|z_{1}\right|+\sigma\left(z^{\prime}\right)<R\right\}$ для $R>0$.

ТЕОрема 3. Пусть $E$ - модельная область конечного типа, а $R(\varepsilon): \mathbb{R} \rightarrow \mathbb{R}^{+} \cup$ $\{+\infty\}-$ такая функиия, что $\lim _{\varepsilon \rightarrow 0} R(\varepsilon)=+\infty$. Пусть $F_{\varepsilon}=E_{\varepsilon} \cap S_{R(\varepsilon)},\left(z_{\varepsilon}, X_{\varepsilon}\right) \in$ $F_{\varepsilon} \times \mathbb{C}^{n} u \lim _{\varepsilon \rightarrow 0}\left(z_{\varepsilon}, X_{\varepsilon}\right)=(z, X) \in E \times \mathbb{C}^{n}$. Тогдa

$$
\lim _{\varepsilon \rightarrow 0} C_{F_{\varepsilon}}^{\infty}\left(z_{\varepsilon}, X_{\varepsilon}\right)=C_{E}^{\infty}(z, X)
$$

СлЕДСТвИЕ 3. Сингулярная метрика Каратеодори модельной области конечного типа непрерывна и совпадает с ее метрикой Азукава.

Кроме того, при условиях теоремы 3 имеем

$$
\lim _{\varepsilon \rightarrow 0} A_{F_{\varepsilon}}\left(z_{\varepsilon}, X_{\varepsilon}\right)=A_{E}(z, X) .
$$


Tеорема 4. Пусть $D$ - гладкая ограниченная псевдовыпуклая область в $\mathbb{C}^{n}$, a $E$ - модельная область $D$ в h-растяжимой граничной точке $p \in \partial D$. Пусть $S$ обозначает любую метрику Каратеодори высшего порядка, или сингулярную метрику Каратеодори, или метрику Азукава. Если X - $(1,0)$ векторное поле, а $\Lambda$ - некасательный конус в $D$ с вершиной в $p$, то

$$
\lim _{\Lambda \ni z \rightarrow p} \frac{S_{D}\left(z, X_{z}\right)}{S_{E}(e, X(z))}=1
$$

равномерно по $X$.

Здесь $e=\left(-1,0^{\prime}\right)$ и

$$
X(z)=\left((-r(z))^{-1}\left(X_{z}\right)_{1},(-r(z))^{-1 / m_{2}}\left(X_{z}\right)_{2}, \ldots,(-r(z))^{-1 / m_{n}}\left(X_{z}\right)_{n}\right),
$$

где $\left(X_{z}\right)_{j}$ есть $j$-я компонента $X_{z}$ в определенных выше локальных координатах.

Предел, указанный в теореме 4 , имеет смысл потому, что модельная область $E$ - гиперболическая по отношению к стандартной метрике Каратеодори [5] (т.е. $C_{E}^{(1)}(z, X)>0$ $\left.\forall z \in E, X \in \mathbb{C}^{n} \backslash\{0\}\right)$.

\section{3. Доказательство результатов.}

ДоКАЗАТЕЛЬСТВо ТЕОРЕмЫ 1 . Поскольку $C_{D}^{(1)}(z, X)$ - непрерьвная функция, гомогенная по второй переменной, из условия теоремы следует, что существуют постоянная $C_{1}>0$ и окрестность $U \Subset D$ точки $z_{0}$ такие, что $C_{D}^{(1)}(z, X) \geqslant C_{1}\|X\| \forall z \in U$, $\forall X \in \mathbb{C}^{n}$. Тогда $\forall z, w \in U, \forall X, Y \in \mathbb{C}^{n}$ имеем

$$
\begin{aligned}
& \left|C_{D}^{(k)}(z, X)-C_{D}^{(k)}(w, Y)\right| \\
& \quad \leqslant\left|\left(C_{D}^{(k)}(z, X)\right)^{k}-\left(C_{D}^{(k)}(w, Y)\right)^{k}\right|\left(C_{1} \max (\|X\|,\|Y\|)\right)^{1-k} .
\end{aligned}
$$

Следовательно, для доказательства теоремы достаточно оценить в отдельности

$$
\left|\left(C_{D}^{(k)}(w, X)\right)^{k}-\left(C_{D}^{(k)}(w, Y)\right)^{k}\right| \text { и }\left|\left(C_{D}^{(k)}(w, X)\right)^{k}-\left(C_{D}^{(k)}(z, X)\right)^{k}\right| .
$$

Предположим, что $C_{D}^{(k)}(w, X) \geqslant C_{D}^{(k)}(w, Y)$. При помоши теоремы Монтеля можно найти экстремальную функцию $f$ для $C_{D}^{(k)}(w, X)$. Тогда в силу неравенства Коши имеем

$$
\begin{aligned}
0 & \leqslant\left(C_{D}^{(k)}(w, X)\right)^{k}-\left(C_{D}^{(k)}(w, Y)\right)^{k} \leqslant\left|f_{(k)}(w) X\right|-\left|f_{(k)}(w) Y\right| \\
& \leqslant \operatorname{dist}^{-k}(U, \partial D) C_{2}\|X-Y\|(\|X\|+\|Y\|)^{k-1}
\end{aligned}
$$

где $C_{2}>0$ - постоянная, зависящая только от $n$ и $k$.

Теперь оценим $\left|\left(C_{D}^{(k)}(z, X)\right)^{k}-\left(C_{D}^{(k)}(w, X)\right)^{k}\right|$.

Обозначим через $C V_{D}(z)$ объем Каратеодори-Эйзенмана $D$ в точке $z \in D$, т.е. $C V_{D}(z)=\sup \operatorname{det}|J F(z)|$, где супремум берется по всем голоморфным отображениям $F$ из $D$ в единичный поликруг в $\mathbb{C}^{n}, F(z)=0$, а $J F=\left(\partial F_{k} / \partial z_{l}\right)_{k, l}$ есть комплексная матрица Якоби $F$. Как упомянуто в $[8], C V_{D}(z)>0$, если только $C_{D}^{(1)}(z, X)>0$ 
$\forall X \in \mathbb{C}^{n} \backslash\{0\}$. Поскольку $C V_{D}(z)$ - непрерьвная функция, имеем $C V_{D}(z) \geqslant C_{3}>0$ $\forall z \in U$.

Пусть $z \in U$ и $F$ - экстремальное отображение для $C V(z)$. Для каждого $l \in \mathbb{N}$, $1 \leqslant l \leqslant n$, положим

$$
\left(\lambda_{l, 1}, \ldots, \lambda_{l, n}\right)=\underbrace{(0, \ldots, 0,1,0, \ldots, 0)}_{l \text {-th place }}(J F(z))^{-1} \text { и } g_{z, l}=\sum_{j=1}^{n} \lambda_{l, j} F_{j} .
$$

Функция $g_{z, l}$ обладает следуюшими свойствами:

$$
g_{z, l}(z)=0, \quad \frac{\partial g_{z, l}}{\partial z_{l}}(z)=1, \quad \frac{\partial g_{z, l}}{z_{j}}(z)=0, \quad 1 \leqslant j \leqslant n, \quad j \neq l .
$$

Кроме того, так как $|\operatorname{det} J F(z)| \geqslant C_{3}>0$ и компоненты $J F(z)$ равномерно ограничены в $U$ (в силу неравенств Коши), имеем $\sup _{D}\left|g_{z, l}\right| \leqslant C_{4} \forall z \in U, 1 \leqslant l \leqslant n$.

Пусть теперь $z, w \in U, X \in \mathbb{C}^{n}, C_{D}^{(k)}(w, X) \geqslant C_{D}^{(k)}(z, X)$, а $f$ - экстремальная функция для $C_{D}^{(k)}(w, X)$. Для каждого мультииндекса $\alpha=\left(\alpha_{1}, \ldots, \alpha_{n}\right)$ положим

$$
h_{z, \alpha}=\frac{1}{\alpha !} \sum_{l=1}^{n}\left(f_{z, l}\right)^{\alpha_{l}} .
$$

Определим следующую последовательность голоморфных функций в $D$ :

$$
f_{0}=f, \quad f_{j+1}=f_{j}-\sum_{|\alpha|=j} \frac{1}{\alpha !} h_{z, \alpha} D^{\alpha} f_{j}(z) .
$$

Поскольку $D^{\alpha} h_{z, \alpha}(z)=1$ и $D^{\beta} h_{z, \alpha}(z)=0 \forall \beta \neq \alpha,|\beta| \leqslant \alpha$, по индукции следует, что $\operatorname{ord}_{z} f_{j} \geqslant j$. С другой стороны, $\left|D^{\alpha} f(z)-D^{\alpha} f(w)\right| \leqslant C_{5}\|z-w\| \forall z, w \in U$ ввиду теоремы о среднем и неравенства Коши. Имеем также $D^{\alpha} f(w)=0$ и $\left|h_{z, \alpha}\right| \leqslant C_{6}$ $\forall|\alpha|<k, \forall z \in U$. Тогда, снова используя теорему о среднем, неравенства Коши и индуктивные аргументы, $\forall z, w \in U, \forall j \leqslant k$ получаем $\left|D^{\alpha} f_{j}(z)\right| \leqslant C_{7}|| z-w \| \forall|\alpha|<k$, $\left|D^{\alpha} f_{j}(z)-D^{\alpha} f(w)\right| \leqslant C_{7}\|z-w\| \forall|\alpha|=k$ и $\sup _{D}\left|f_{j}\right| \leqslant 1+C_{7}\|z-w\|$. Из этого и из определения $C_{D}^{(k)}(w, X)$ следует, что

$$
\left(1+C_{6}\|z-w\|\right)\left(C_{D}^{(k)}(z, X)\right)^{k} \geqslant \mid\left(f_{(k)}(z) X|\geqslant| f_{(k)}(w) X \mid-C_{7}\|X\|^{k}\|z-w\| .\right.
$$

Ввиду того, что $C_{D}^{(k)}(z, X) \leqslant\|X\| \operatorname{dist}^{-1}(z, \partial D)$, a $f$ - экстремальная для $C_{D}^{(k)}(w, X)$, заключаем, что

$$
0 \leqslant\left(C_{D}^{(k)}(w, X)\right)^{k}-\left(C_{D}^{(k)}(z, X)\right)^{k} \leqslant C_{8}\|X\|^{k}\|z-w\| .
$$

Неравенства (1), (2) и (3) завершают доказательство теоремы 1. 
ДокАЗАТЕЛЬСТВо ТЕОРЕМЫ 2. Пользуясь неособым линейным отображением (если это необходимо), можно предположить, что компоненты вектора $X$ не равны 0, а значит, и компоненты векторов $X_{j} \rightarrow X$ тоже не равны 0. Рассмотрим линейные отображения $\Psi_{j}(w)=\frac{\left(w-z_{j}\right) X}{X_{j}}+z$ и положим $G_{j}=\Psi_{j}\left(D_{j}\right)$. Имеем

$$
C_{D_{j}}^{\infty}\left(z_{j}, X_{j}\right)=C_{G_{j}}^{\infty}(z, X)
$$

Поскольку $D$ - строго гипервьпуклая область, существуют другая область $D^{\prime} \ni D$ и плюрисубгармоническая функция $\varphi$ в $D^{\prime}$ такие, что $\lim _{z \rightarrow \partial D} \varphi(z)=0$ и $D=\left\{z \in D^{\prime}\right.$ : $\varphi(z)<0\}$. Пусть $\varepsilon>0$ таково, что открытое множество $\left\{w \in D^{\prime}: \varphi(w)<-\varepsilon\right\}$ содержит точку $z$. Обозначим через $D_{\varepsilon}$ связную компоненту этого множества, содержащую $z$. Пусть $R>0$ такова, что шар $B(0, R) \ni D$, а $D_{-\varepsilon}-$ связная компонента открытого множества $B(z, R) \cap\left\{w \in D^{\prime}: \varphi(w)<\varepsilon\right\}$, содержащая $D$. Поскольку $D_{\varepsilon} \Subset D \Subset D_{-\varepsilon}$ $\forall j \gg 1$, найдутся области $G_{\varepsilon}$ и $G_{-\varepsilon}$ такие, что $D_{\varepsilon} \Subset G_{\varepsilon} \subset G_{j} \subset G_{-\varepsilon} \Subset D_{-\varepsilon}$ и $G_{\varepsilon} \subset D \subset G_{-\varepsilon}$. Из этого следует, что

$$
\begin{aligned}
& C_{G_{-\varepsilon}}^{\infty}(z, X) \leqslant C_{G_{j}}^{\infty}(z, X) \leqslant C_{G_{\varepsilon}}^{\infty}(z, X) \\
& C_{G_{-\varepsilon}}^{\infty}(z, X) \leqslant C_{D}^{\infty}(z, X) \leqslant C_{G_{\varepsilon}}^{\infty}(z, X) .
\end{aligned}
$$

Докажем, что

$$
\lim _{\varepsilon \rightarrow 0}\left(C_{G_{\varepsilon}}^{\infty}(z, X)-C_{G_{-\varepsilon}}^{\infty}(z, X)\right)=0
$$

что вместе с (4), (5) и (6) завершит доказательство теоремы 2.

Обозначим через $g_{-\varepsilon}$ функцию Грина $D_{-\varepsilon}$. Пусть $\chi-C^{\infty}$-гладкая функция такая, что $\chi \equiv 1$ в $D_{\varepsilon}$ и $\operatorname{supp} \chi \subset G_{\varepsilon}$. Пусть $m \in \mathbb{N}$ и $f_{m}$ - экстремальная функция для $C_{G_{\varepsilon}}^{(m)}(z, X)$. Поскольку $g_{-\varepsilon}(z, \cdot)$ - плюрисубгармоническая функция и $g_{-\varepsilon}(z, w)>-\infty$, $z \neq w$ (ибо $D_{-\varepsilon}$ ограничена), можно решить $\bar{\partial}$-проблему $\bar{\partial} h_{m}=\bar{\partial}\left(\chi f_{m}\right)$ в ограниченной псевдовьпуклой области $D_{-\varepsilon}(0<\varepsilon \ll 1)$ с $L^{2}$ оценкой [9]

$$
\begin{aligned}
\int_{D_{-\varepsilon}}\left|h_{m}\right|^{2} \frac{\exp \left(-2(m+n) g_{-\varepsilon}(z, w)\right)}{\left(1+\|w\|^{2}\right)^{2}} d V(w) \\
\quad \leqslant 2 \int_{D_{-\varepsilon}}\left|\bar{\partial}\left(\chi f_{m}\right)\right|^{2} \exp \left(-2(m+n) g_{-\varepsilon}(z, w)\right) d V(w) .
\end{aligned}
$$

Очевидно, что $t_{m}=\chi f_{m}-h_{m}$ - голоморфная функция в $D_{-\varepsilon}$. Из сходимости первого интеграла и из неравенства

$$
g_{-\varepsilon}(z, w) \leqslant \log \frac{\|w-z\|}{\operatorname{dist}\left(z, \partial D_{-\varepsilon}\right)}
$$

[1] следует, что $\operatorname{ord}_{z} h_{m} \geqslant m+1$. Тогда, пользуясь тем, что $\chi \equiv 1$ в $D_{\varepsilon} \ni z$, получаем

$$
\left(t_{m}\right)_{(m)}(z) X=\left(f_{m}\right)_{(m)}(z) X
$$


Теперь оценим $\sup _{G_{-\varepsilon}}\left|t_{m}\right|$. Из неравенства о среднем следует, что

$$
\begin{gathered}
\operatorname{Vol} B(O, 1)\left(\operatorname{dist}\left(G_{-\varepsilon}, \partial D_{-\varepsilon}\right)\right)^{2 n} \sup _{G_{-\varepsilon}}\left|t_{m}\right|^{2} \leqslant \int_{D_{-\varepsilon}}\left|t_{m}\right|^{2} \\
\leqslant 2 \int_{D_{-\varepsilon}}\left(\left|\chi f_{m}\right|^{2}+\left|h_{m}\right|^{2}\right) \leqslant 2\left(\operatorname{Vol} G_{\varepsilon}+\int_{D_{-\varepsilon}}\left|h_{m}\right|^{2}\right) .
\end{gathered}
$$

Поскольку $g_{-\varepsilon}$ отрищательна в $D_{-\varepsilon}$, из $(8)$ получаем, что

$$
\int_{D_{-\varepsilon}}\left|h_{m}\right|^{2} \leqslant 2\left(1+R^{2}\right)^{2} \max _{w \in \operatorname{supp} \bar{\partial} \chi} \exp \left(-2(m+n) g_{-\varepsilon}(z, w)\right) \int|\bar{\partial} \chi|^{2} .
$$

С другой стороны, так как $D_{-\varepsilon} \subset B(0, R)$, имеем, что (см. [6])

$$
g_{-\varepsilon}(z, w) \geqslant C \sup _{k \in \mathbb{N}}\left(k(\varphi(w)-\varepsilon)+\frac{\|w\|^{2}-R^{2}}{k}\right)
$$

для $w$ вне окрестности $U$ точки $z$, где постоянная $C$ зависит только от диаметра $U$ и $R$. Поскольку $\operatorname{supp} \bar{\partial} \chi \subset G_{\varepsilon} \backslash D_{\varepsilon} \subset D_{-\varepsilon} \backslash D_{\varepsilon}$, имеем

$$
\max _{w \in \operatorname{supp} \bar{\partial} \chi} \exp \left(-2(m+n) g_{-\varepsilon}(z, w)\right) \leqslant b(\varepsilon, m)
$$

где $b(\varepsilon, m)=\inf _{k \in \mathbb{N}} \exp \left(2 C(m+n)\left(2 k \varepsilon+\frac{R^{2}}{k}\right)\right)$. Вместе с (10) и (11) это показьвает, что

$$
\sup _{G_{-\varepsilon}}\left|t_{m}\right| \leqslant C_{\varepsilon}(1+b(\varepsilon, m))
$$

для какой-то постоянной $C_{\varepsilon}$, не зависящей от $m \in \mathbb{N}$.

Поскольку $f_{m}-$ экстремальная функция для $C_{G_{\varepsilon}}^{(m)}(z, X)$, можно заключить ввиду определения $C_{G_{-\varepsilon}}^{(m)}(z, X),(9)$ и $(12)$, что

$$
C_{G_{-\varepsilon}}^{m}(z, X) \geqslant C_{G_{\varepsilon}}^{m}(z, X)\left(C_{\varepsilon}(1+b(\varepsilon, m))\right)^{\frac{1}{m}} .
$$

Переходя последовательно к пределам при $m \rightarrow \infty, \varepsilon \rightarrow 0+, k \rightarrow \infty$ и используя (6), получаем (7).

ДокАЗАТЕЛЬСТво СЛЕДСТВИЯ 2. В силу результата Нивоша [2] для каждой точки $z \in D$ имеем $C_{D}^{\infty}(z, X)=A_{D}(z, X)$ для почти всех $X \in \mathbb{C}^{n}$. С другой стороны, $A_{D}(z, \cdot)$ - логарифм-плюрисубгармоническая функция [10]. Непрерьвная функция $C_{D}^{\infty}(z, \cdot)$ также логарифм-плюрисубгармонична в качестве супремума таких функций $\left\{C_{D}^{k}(z, \cdot)\right\}_{k=1}^{\infty}$. Известно, что если две плюрисубгармонические функции совпадают почти всюду, то они совпадают всюду. Поэтому $C_{D}^{\infty}(z, X)=A_{D}(z, X)$ в $D \times \mathbb{C}^{n}$.

Из этого и из неравенства $A_{D}\left(z_{j}, X_{j}\right) \geqslant C_{D_{j}}^{\infty}\left(z_{j}, X_{j}\right)$ следует, что

$$
\liminf _{j \rightarrow \infty} A_{D}\left(z_{j}, X_{j}\right) \geqslant A_{D}(z, X) .
$$

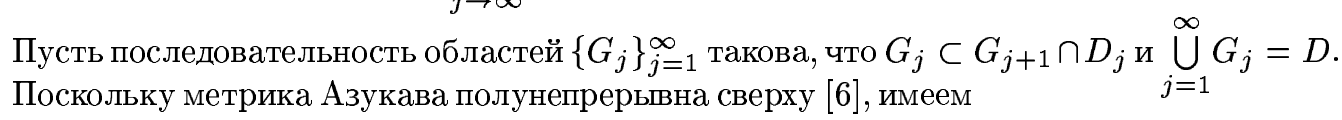

$$
\limsup _{j \rightarrow \infty} A_{D_{j}}\left(z_{j}, X_{j}\right) \leqslant \limsup _{j \rightarrow \infty} A_{G_{l}}\left(z_{j}, X_{j}\right) \leqslant A_{G_{l}}(z, X)
$$

$\forall l \in \mathbb{N}$. C другой стороны, $\lim _{l \rightarrow \infty} A_{G_{l}}(z, X)=A_{D}(z, X)[10]$, что вместе с (13) и (14) дает $\lim _{j \rightarrow \infty} A_{D}\left(z_{j}, X_{j}\right)=A_{D}(z, X)$. 
ДокАЗАТЕЛЬСТво тЕоРЕмы 3. Доказательство теоремы 3 аналогично доказательству теоремы 2 .

В силу результатов [3], [7] модель $E$ - область конечного типа, если только существует гладкая положительная функция $a$ в $\mathbb{C}^{n-1} \backslash\{0\}$ такая, что

$$
a\left(t^{1 / m_{2}} z_{2}, \ldots, t^{1 / m_{n}} z_{n}\right)=t a\left(z^{\prime}\right) \quad \forall t>0, \quad z^{\prime} \in \mathbb{C}^{n-1} \backslash\left\{0^{\prime}\right\}
$$

и $P-2 a-$ строго плюрисубгармоническая функция в $\mathbb{C}^{n-1} \backslash\left\{0^{\prime}\right\}$. Можно считать, что $a\left(0^{\prime}\right)=0$. Для $\forall \varepsilon \in(0,1]$ положим

$$
G_{-\varepsilon}=\left\{z \in \mathbb{C}^{n}: r_{-\varepsilon}(z):=\operatorname{Re} z_{1}+P\left(z^{\prime}\right)-\varepsilon\left(1+a\left(z^{\prime}\right)\right)<0\right\}
$$

Поскольку $F_{\varepsilon}=E_{\varepsilon} \cap S_{R(\varepsilon)}(\varepsilon \geqslant 0)$ - строго гипервьпуклая область, достаточно доказать, в силу теоремы 2 , что

$$
\lim _{\varepsilon \rightarrow 0+}\left(C_{G_{-\varepsilon / 2}}^{\infty}(z, X)-C_{F_{\varepsilon}}^{\infty}(z, X)\right)=0
$$

равномерно на компактных подмножествах $E \times \mathbb{C}^{n}$.

Обозначим через $g_{-\varepsilon}$ функцию Грина $G_{-\varepsilon}$. Поскольку алгебра ограниченных голоморфных функций в $G_{-\varepsilon}$ разделяет точки $G_{-\varepsilon}[3]$, имеем

$$
g_{-\varepsilon}(z, w)>-\infty, \quad z \neq w
$$

С другой стороны, $C_{G_{-1}}^{(1)}(z, X)>0 \forall z \in G_{-1}, X \in \mathbb{C}^{n}$ [5]. Из этого следует, что если $f$ - экстремальная функция для $C_{G_{-1}}^{(1)}(z, X)$, то форма Леви $|f|^{2}$ положительна вблизи $(z, X)$. Теперь, пользуясь стандартными аргументами, для каждого компактного подмножества $K \subset G_{-1}$ можно построить гладкую ограниченную плюрисубгармоническую функцию в $G_{-1}$, которая строго плюрисубгармонична в $K$.

Пусть $K$ - компактное подмножество $E$ и $H_{\varepsilon}=E_{2 \varepsilon} \cap S_{R(\varepsilon) / 2}$. Для $0<\varepsilon \ll 1$ имеем $K \Subset H_{\varepsilon} \Subset F_{\varepsilon} \Subset G_{-\varepsilon / 2} \Subset G_{-\varepsilon}$. Пусть $\chi-$ гладкая функция такая, что $\chi \equiv 1$ в $H_{\varepsilon}$ и $\operatorname{supp} \chi \subset F_{\varepsilon}$. Пусть $(z, X) \in K \times \mathbb{C}^{n}, m \in \mathbb{N}$, а $f_{m}$ - экстремальная функция для $C_{F_{\varepsilon}}^{(m)}(z, X)$. Как мы уже установили, существует гладкая плюрисубгармоническая функция $s$ в $G_{-1},-1 \leqslant s \leqslant 0$, которая строго плюрисубгармонична в supp $\chi$. Обозначим через $c$ наименьшее из собственных значений формы Леви $s$ в supp $\chi$. Ввиду (17) можно решить $\bar{\partial}$-задачу $\bar{\partial} h_{m}=\bar{\partial}\left(\chi f_{m}\right)$ в псевдовыпуклой области $G_{-\varepsilon}$ с $L^{2}$-оценкой [9]:

$$
\begin{aligned}
& \int_{G_{-\varepsilon}}\left|h_{m}\right|^{2} \exp \left(-2(m+n) g_{-\varepsilon}(z, w)-s\right) d V(w) \\
& \quad \leqslant c^{-1} \int_{G_{-\varepsilon}}\left|\bar{\partial}\left(\chi f_{m}\right)\right|^{2} \exp \left(-2(m+n) g_{-\varepsilon}(z, w)-s\right) d V(w) .
\end{aligned}
$$

Тогда $t_{m}=\chi f_{m}-h_{m}$ - голоморфная функция в $G_{-\varepsilon}$. Нетрудно видеть, что $\mid P\left(z^{\prime}+\delta\right)-$ $P\left(z^{\prime}\right)|\leqslant C| \delta\left|\left(1+\sigma\left(z^{\prime}\right)\right) \forall \delta,\right| \delta \mid \leqslant 1$, из чего следует, что $\operatorname{dist}\left(G_{-\varepsilon / 2}, \partial G_{-\varepsilon}\right)>0$. 
Как и в доказательстве теоремы 2 , получаем

$$
\begin{gathered}
\left(t_{m}\right)_{(m)}(z) X=\left(f_{m}\right)_{(m)}(z) X \\
\sup _{G_{-\varepsilon / 2}}\left|t_{m}\right| \leqslant C_{\varepsilon}\left(1+\sup _{w \in F_{\varepsilon} \backslash H_{\varepsilon}} \exp \left(-2(m+n) g_{-\varepsilon}(z, w)\right)\right) .
\end{gathered}
$$

Итак, чтобы получить (16), достаточно доказать, что

$$
\lim _{\varepsilon \rightarrow 0+} \inf _{w \in F_{\varepsilon} \backslash H_{\varepsilon}}\left(\exp g_{-\varepsilon}(z, w)\right)^{-2(m+n)}=0
$$

равномерно по $z \in K$.

Поскольку существует плюрисубгармоническая функция $q_{\text {в }} G_{-1} \supset G_{-\varepsilon},-1 \leqslant q \leqslant 0$, которая строго плюрисубгармонична в $K$, имеем [1]

$$
\begin{gathered}
g_{-\varepsilon}(z, w) \geqslant C \sup _{k \in \mathbb{N}}\left(k r_{-\varepsilon}(w)+\frac{q(w)}{k}\right) \\
\forall z \in K \subset E, \quad w \in G_{-\varepsilon} \backslash H_{\varepsilon} \supset F_{\varepsilon} \backslash H_{\varepsilon} \ni K .
\end{gathered}
$$

В силу определений $E$ (см. раздел 2$)$ и $G_{-\varepsilon}$ (см. (15)) получаем

$$
g_{-\varepsilon}(z, w) \geqslant-C \inf _{k \in \mathbb{N}}\left(k \varepsilon\left(1+a\left(w^{\prime}\right)\right)+\frac{1}{k}\right) \quad \forall z \in K, \quad w \in F_{\varepsilon} \backslash H_{\varepsilon} .
$$

Пусть $g$ обозначает функцию Грина области $G=\left\{z \in \mathbb{C}^{n}: \operatorname{Re} z_{1}+P\left(z^{\prime}\right)-a\left(z^{\prime}\right)<0\right\}$. Поскольку $g_{-\varepsilon}(z, w) \geqslant g(z-1, w)$, равенство (18) будет следствием (19), если

$$
\lim _{w \in G ; w \rightarrow \infty} g(z, w)=0
$$

равномерно выполнено по $z \in G \cap S(R)$ для произвольного числа $R>0$.

Чтобы доказать (20), воспользуемся тем, что существует функция пика $G$ в граничной точке 0 [3], т.е. голоморфная функция $p$ в $G$, непрерывная в $\bar{G}$ и такая, что $p(0)=1$ и $|p(\zeta)|<1$ в $\bar{G} \backslash\{0\}$. Положим $\pi_{t}(\zeta)=\left(t^{-1} \zeta_{1}, t^{-1 / m_{2}} \zeta_{2}, \ldots, t^{-1 / m_{n}} \zeta_{n}\right), t>0$, $v_{w}(\zeta)=p\left(\pi_{\sigma(w)}(\zeta)\right), u_{w}(\zeta)=\log \left|\left(v_{w}(z)-v_{w}(\zeta)\right) /\left(1-\bar{v}_{w}(z) v_{w}(\zeta)\right)\right|$. Поскольку $\pi_{t}$ - автоморфизм $G$, а $p$ - функция пика $G$ в 0 , то $u_{w}(\zeta)$ - отрицательная плюрисубгармоническая функция в $G$ с особенностью типа $\log |\zeta-z|$ в точке $z$. В силу определения $S_{R}$ (см. раздел 2) мы получаем, что $\lim _{w \in G ; w \rightarrow \infty} u(z, w)=0$ равномерно по $z \in G \cap S(R)$. Из этого следует (20), чем завершается доказательство теоремы 3.

Следствие 3 следует из теоремы 3 , следствия 2 и того факта, что области $E \cap S_{R}$ строго гипервьпуклые. 
ДоКАЗАТЕЛЬСТво ТЕОРЕмЫ 4. Случай стандартной метрики Каратеодори доказан в [5].

Поскольку модельная область $E$ - гиперболическая по отношению к стандартной метрике Каратеодори [5], из теоремы 1 следует, что метрики Каратеодори высшего порядка непрерывны в $E \times \mathbb{C}^{n}$. Тогда те же самые аргументы, что и в [5], дают теорему 4 для любой из этих метрик.

Из этого следует, что

$$
\liminf _{\Lambda \ni z \rightarrow p} \frac{C_{D}^{\infty}\left(z, X_{z}\right)}{C_{E}^{\infty}(e, X(z))} \geqslant 1 .
$$

Предположим противное. Тогда найдутся векторное поле $X$ и последовательность точек $\Lambda \ni\left\{z_{j}\right\}_{j=1}^{\infty} \rightarrow p$ такие, что $\lim _{\Lambda \ni z_{j} \rightarrow p}\left(X\left(z_{j}\right) /\left\|X\left(z_{j}\right)\right\|\right)=Y$ и

$$
\liminf _{\Lambda \ni z_{j} \rightarrow p} \frac{C_{D}^{\infty}\left(z_{j}, X_{z_{j}}\right)}{C_{E}^{\infty}\left(e, X\left(z_{j}\right)\right)}<1
$$

Поскольку $C_{E}^{\infty}-$ непрерьвная функция (следствие 3 ), можно заключить, что

$$
\liminf _{\Lambda \ni z_{j} \rightarrow p} \frac{C_{D}^{\infty}\left(z_{j}, X_{z_{j}}\right)}{\left\|X\left(z_{j}\right)\right\|}<C_{E}^{\infty}(e, Y)
$$

Следовательно,

$$
\liminf _{\Lambda \ni z_{j} \rightarrow p} \frac{C_{D}^{(k)}\left(z_{j}, X_{z_{j}}\right)}{\left\|X\left(z_{j}\right)\right\|}<C_{E}^{(k)}(e, Y)
$$

$\forall k \gg 1$, а это противоречие.

Поскольку $C_{D}^{\infty} \leqslant A_{D}$ и $C_{E}^{\infty} \equiv A_{E}$ (следствие 3 ), чтобы завершить доказательство теоремы 4 , достаточно доказать неравенство

$$
\limsup _{\Lambda \ni z \rightarrow p} \frac{A_{D}\left(z, X_{z}\right)}{A_{E}(e, X(z))} \leqslant 1 .
$$

Пусть $\varepsilon>0$ произвольно. В силу определения $E_{\varepsilon}$ и $S_{\delta}$ (см. раздел 2) существует такое число $\delta=\delta(\varepsilon)>0$, что $E_{\varepsilon} \cap S_{\delta} \subset D$. Поскольку $\pi_{-r(z)}-$ автоморфизм $E$ и $X(z)=\pi_{-r(z)}\left(X_{z}\right)$, имеем

$$
A_{D}\left(z, X_{z}\right) \leqslant A_{F_{z, \varepsilon}}(e(z), X(z))
$$

где $e(z)=\pi_{z}(z)$ и $F_{z, \varepsilon}=E_{\varepsilon} \cap U_{-\delta / r(z)}$. Очевидно, что $\lim _{z \rightarrow p}(-\delta / r(z))=+\infty$ и $\lim _{\Lambda \ni z \rightarrow p} e(z)=\left(-1,0^{\prime}\right)=e$. Тогда теорема 3 дает

$$
\lim _{\Lambda \ni z \rightarrow p} \frac{A_{F_{z, \varepsilon}}(e(z), X(z))}{A_{E_{\varepsilon}}(e, X(z))}=1, \quad \lim _{\varepsilon \rightarrow 0+} \frac{A_{E \varepsilon}(e(z), X(z))}{A_{E}(e, X(z))}=1 .
$$

Теперь (21) следует из (22) и (23). 


\section{СПИСОК ЦИТИРОВАННОЙ ЛИТЕРАТУРЫ}

[1] Jarnicki M., Pflug P. Invariant distances and metrics in complex analysis. Berlin, New York: De Gruyter, 1993.

[2] Nivoche St. The pluricomplex Green function, capacitative notions, and approximation problems in $\mathbb{C}^{n}$ // Indiana Univ. Math. J. 1995. V. 44. P. 489-510.

[3] Yu J. Peak functions on weakly pseudoconvex domains // Indiana Univ. Math. J. 1994. V. 43. P. 1271-1295.

[4] Zwonek W. Regularity Properties of the Azukawa Metric. Preprint.

[5] Nikolov N. Nontangential weighted limit of the infinitesimal Caratheodory metric in an $h$-extendible boundary point of a smooth bounded pseudoconvex domain in $\mathbb{C}^{n} / /$ Acta Math. Hung. 1999. V. 82. P. 311-324.

[6] Jarnicki M., Pflug P. The pluricomplex Green function // Indiana Univ. Math. J. 1995. V. 44. P. 535-543.

[7] Diederich K., Herbort G. Pseudoconvex domains of semiregular type // Contributions to Complex Analysis and Analytic Geometry / ed. H. Skoda and J. M. Trepreau. Braunschweig: Aspects of Math. E26. Vieweg, 1994. P. 127-162.

[8] Полецкий Е. А., Шабат Б. В. Инвариантные метрики // Итоги науки и техники. Современные проблем математики. Фундаментальные направления. Т. 9. М.: ВИНИТИ АН СССР, 1986. C. $73-125$.

[9] Hörmander L. $L^{2}$ estimates and existence theorems for $\bar{\partial}$ operator // Acta Math. 1965. V. 113. P. 89-152.

[10] Azukawa K. The invariant pseudo-metric related to negative plurisubharmonic functions // Kodai Math. J. 1987. V. 10. P. 83-92.

Институт математики и информатики Болгарской академии наук, 